\title{
Repetition blindness: Type recognition without token individuation*
}

\author{
NANCY G. KANWISHER
}

Harvard University

\begin{abstract}
Three experiments are described which use RSVP (rapid serial visual presentation) to demonstrate a new cognitive phenomenon called "repetition blindness". Subjects have difficulty detecting repeated words-even when the two occurrences are nonconsecutive and differ in case (Experiment 1). In immediate verbatim recall of sentences (Experiment 2), subjects selectively omitted second instances of repeated words, sacrificing the meaning and grammaticality of the sentence. In Experiment 3, recognition threshold for the last word in a list was lowered, not elevated, when that word had also occurred earlier in the same list. Thus, repetition blindness does not result from a refractory period for recognition of second occurrences. These findings support a distinction between the perceptual processes of (i) recognizing a word as being of a certain type, and (ii) individuating a word as a particular token of that type: repetition blindness occurs when words are recognized as types but not individuated as tokens.
\end{abstract}

\section{Introduction}

Much of the recent work on visual perception has addressed the problem of recognition: how are stimuli categorized as instances of certain stored visual types? What has been largely ignored, however, is the question of how we distinguish between different instances, or tokens, of the same type. ${ }^{1}$ Such information is nevertheless crucial for certain basic visual tasks. Suppose a

\footnotetext{
*I thank Mary Potter for her invaluable help with the research and comments on the manuscript. I also thank Audra Noel for research assistance, and the following people for comments on this and earlier versions of the manuscript: Hiram Brownell, Susan Carey, Steve Pinker, John Rubin, Janelle Schweickert, Ed Smith, and two anonymous reviewers. This work was supported by NSF grant BNS83-18156 to Mary C. Potter.

Reprint requests should be addressed to: N.G. Kanwisher, Department of Psychology, Harvard University, 33 Kirkland Street, Cambridge, MA 02138, U.S.A.
} 
viewer is looking at a bowl of fruit. Consider how he might answer the question, how many pears are there? Type information alone-that there are pears in the bowl-is insufficient. The viewer must determine how many particular tokens there are of the given type. Similarly, to select the reddest apple, the viewer will need to compare particular apples. Type information alone would therefore be insufficient for either task, as it would merely indicate the presence of (for example) apples, pears, bananas, and the feature type, redness. To count the pears, or determine if a particular apple is red, one must effectively label or in some way individuate each item of a given type to distinguish it from other items of the same type.

Distinguishing between different tokens of the same type is also necessary to understand language. Consider the different meanings of the sentences "Big fish eat little fish" and "Big fish eat little". To understand the first sentence, and to clearly distinguish it from the second, both instances of the word "fish" must be recognized and encoded as two different tokens. A simple list of the word types that occurred in the sentence would be insufficient, as it would be the same for both sentences. Thus, the ability to separately encode different tokens of the same type is crucial to both visual perception and language understanding. Under everyday conditions, people are adept at encoding such information. Sentences with repeated words, and visual arrays with several tokens of the same type, seem to present little difficulty to the perceiver.

By contrast, the current experiments demonstrate conditions under which subjects recognize types but fail to separately encode different tokens of the same type. Consider the task of determining whether a list of words displayed in rapid serial visual presentation (RSVP) contains any repated words. At rates of presentation slower than about 5 words per second, this is a natural and very easy task: subjects detect almost all repetitions (Kanwisher, 1986). However, repetition detection becomes surprisingly difficult at presentation rates which are faster than normal reading or spcaking, yct which allow easy recognition of most of the items presented. This phenomenon, first noticed by $\mathrm{H}$. Intraub and $\mathrm{M}$. Potter while carrying out pilot work on a different problem (personal communication, 1984), will be called "repetition blindness".

Repetition blindness contrasts markedly with earlier work on the effects of repetition, which generally involve a benefit for repeated as compared to unrepeated words. Past work has indicated both that repeated words are encoded more quickly than unrepeated ones (e.g., Scarborough, Cortese, \& Scarborough, 1977), and that subjects are normally adept at separately encoding different occurrences of the same word (Hintzman \& Block, 1971). Thus, repetition blindness does not fit with and cannot be explained by past work 
on repetitions. A full account of the phenomenon is therefore likely to teach us something new about visual information processing.

It will be argued here that repetition blindness occurs when words are recognized as types but not individuated as separate tokens. This account will be called the "Token Individuation" Hypothesis. Before this hypothesis is to be taken seriously, however, several alternative accounts of repetition blindness must be considered. The experiments described here will evaluate four alternative accounts of the failure to detect repetitions at rapid presentation rates (see Table 1).

First, the Recognition Failure Hypothesis holds that subjects fail to detect repetitions because at high rates of presentation they simply cannot recognize all the words-repeated or not. On this view, there is no particular difficulty in encoding or noticing repetitions of words. Rather, failure at the task derives from the general difficulty of recognizing rapidly-presented words.

According to the Forgetting Hypothesis, the words are adequately recognized but the first occurrence of the repeated word (henceforth "R1") is forgotten before the second occurrence ("R2") appears. As it has been shown that rapidly-presented words can be recognized and quickly forgotten (Potter, 1984), it is not clear whether subjects can remember R1 at least until R2 appears. Thus, like the Recognition Failure Hypothesis, the Forgetting Hypothesis explains repetition blindness not in terms of a particular difficulty encoding repeated words, but in terms of a general difficulty in processing rapidly-presented words.

The Multiple Comparisons Hypothesis, in contrast, holds that the list words are easily recognized and remembered. However, according to this hypothesis the subject must compare each successive word with the words which have already appeared, in order to determine whether it is a repetition. This may require more comparisons than the time per word permits, and the subject may be unable to complete the task before the next word appears. If the unfinished comparison process is cut off when the next list word appears,

Table 1. The different hypotheses accounting for repetition blindness, and their likelihood based on Experiments 1 through 3

\begin{tabular}{llll}
\hline Hypothesis & Exp. 1 & Exp. 2 & Exp. 3 \\
\hline Recognition Failure Hypothesis & Unlikely & No & No \\
Forgetting Hypothesis & No & No & No \\
Multiple Comparisons Hypothesis & Possible & No & No \\
Refractory Period Hypothesis & Possible & Possible & No \\
Token Individuation Hypothesis & Possible & Possible & Yes \\
\hline
\end{tabular}


repetitions may go unnoticed. Thus, the Multiple Comparisons Hypothesis explains repetition blindness in terms of the difficulty of determining which of two recognized list words are the same.

According to the Refractory Period Hypothesis, the first occurrence of the repeated word causes the recognition unit for that word to become refractory, raising the recognition threshold for that word. If the second occurrence, R2, appears during this refractory period, the subject would have difficulty recognizing it and might be unaware of the repetition. An example of a similar elevation of detection threshold is seen in the case of visual aftereffects. After observing a vertical grating, for example, it is more difficult to detect a second faint vertical grating (Gilinsky, 1968). Such aftereffects are usually explained as the result of fatigued detectors. It is an interesting possibility, therefore, that word recognition elements may become similarly fatigued. If so, this might result in elevation of the recognition threshold for the repeated word such that sometimes R2 would not be recognized and the repetition would not be detected.

The experiments reported here eliminate these four alternative hypotheses and support the Token Individuation hypothesis. A model for repetition blindness and token individuation will be presented and discussed in the context of other related cognitive phenomena.

\section{Experiment 1}

Experiment 1 was designed to demonstrate the phenomenon of repetition blindness and to characterize its temporal properties. Subjects' ability to report which word was repeated within rapid serial lists was measured. Two parameters of the list presentation were varied: (i) lists were presented at different rates, and (ii) words were repeated at different lags within the list. The lag was simply the number of words intervening between the first instance (R1) and second instance (R2) of the repeated word.

In order to control reading rate, rapid serial visual presentation (RSVP) was used for all the experiments in this study. Earlier work (Potter, 1984) has shown that RSVP reading is quite natural and that sentential material can be accurately read at rates up to 12 words per second.

\section{Method}

\section{Subjects}

Forty subjects were recruited for this experiment. They were native speakers of American English from the MIT community, and were under 30 years 
of age. They were paid for their participation. (This description applies to all subjects used in the experiments reported here.)

\section{Materials and design}

Thirty lists of 7 nouns were constructed by sampling randomly without replacement from a pool of nouns which were 5,6 , or 7 letters long. One target word was chosen randomly from each list, to occur in two different serial positions (R1 and R2). There were therefore eight word presentations in each list. R1 was capitalized and R2 was in lower case in half of the lists; the reverse was true for the other half of the lists. The order of the remaining six words, which were half in capital letters and half in lower case, was randomized.

To vary lag while controlling for serial position effects, this experiment used 10 versions of each list of words, counterbalanced over 10 versions of the experiment. Each of the 10 versions of the experiment contained 3 lists in each of the 10 list conditions, making 30 lists per version. In conditions 1 through 4, the serial position of R1 was always 2 , and R2 occupied serial positions $4,5,6$, or 7 , respectively. In conditions $5-8$, the serial position of R2 was always 7 , while R1 occurred in positions $5,4,3$, or 2, respectively. Finally, in conditions 9 and 10 (which were identical, for symmetry of design), $\mathrm{R} 1$ was the first word in the list and $\mathrm{R} 2$ was the last. The inclusion of the last two conditions was expected to provide an upper bound on repetition detectability. Serial position was therefore counterbalanced in the following way. For conditions 1 through 4 (with R1 always in serial position 2), the serial position of R2 was positively correlated with distance. For conditions 5-8 (with $\mathrm{R} 2$ always in serial position 7), the serial position of R1 was negatively correlated with distance. Thus, in the analysis of conditions 1-8 together, serial position effects should generally cancel, leaving only lag effects.

The experiment was run at four different rates: words were presented for $117,150,183$, or $250 \mathrm{~ms}$. (There was no interval between the presentation of successive words.) There was one subject in each version of the experiment for each rate condition.

\section{Procedure}

Three practice trials preceded the main experiment. Each trial began when the subject pressed the space bar. A row of asterisks appeared on the computer screen for $500 \mathrm{~ms}$ at the same location as the subsequent words. Then the list appeared, one word at a time in the same place, followed by a 250 ms mask (a row of six percent signs).

As soon as the list ended, subjects named the word they thought had occurred twice in the list, guessing if unsure. Then they gave a confidence 
rating for their response-_" 1 " if they were sure, "2" if they thought they were probably correct, and " 3 " if they were just guessing.

\section{Apparatus}

In this and all experiments reported here a Terak microcomputer was used to present the stimuli on a CRT screen with a rapid fade phosphor. The subjects' response was recorded by the experimenter.

\section{Results}

The accuracy of repetition detection as a function of lag was scored by combining data from the two conditions with the same R1-R2 distance, thus controlling for serial position as described above. For example, condition 1 (serial positions 2 and 4 ) was combined with condition 5 (serial positions 5 and 7 ). The average percent of correct repetition detections for each lag and rate is shown in Table 2. All statistical analyses excluded the lag 6 condition, since repeated words in this condition appeared first and last in their lists. A subject analysis showed significant main effects of lag, $F(3,108)=3.0, p<$ .05 , and rate, $F(3,36)=16.1, p<.0001$. There was no significant interaction of Lag $\times$ Rate, $F<1.0$. The overall increase in repetition detectability with increasing lag was significant in a linear trend analysis, $F(1,108)=10.8, p$ $<.01$. In separate analyses of each rate, the trend analysis was significant for the $117 \mathrm{~ms}$ rate, $p<.05$, and marginally significant for the $150 \mathrm{~ms}$ rate, $p<$ .1 , but not significant for the two slower rates. Confidence ratings gave the same picture: of the correct responses in Table 2 , subjects were more confident of their responses at larger lags and slower rates (see Table 3 ).

Table 2. Percentage of repetition detections as a function of lag and rate (ms/word), Experiment 1

\begin{tabular}{lllllll}
\hline & & \multicolumn{2}{c}{ Lag } & & & \\
\hline & 1 & 2 & 3 & 4 & $6^{\mathrm{a}}$ & Mean $^{\mathrm{b}}$ \\
\hline 117 & 35 & 43 & 48 & 55 & 83 & 45 \\
150 & 50 & 57 & 60 & 65 & 87 & 58 \\
183 & 65 & 62 & 72 & 65 & 82 & 66 \\
250 & 90 & 88 & 95 & 95 & 90 & 91 \\
Mean & 60 & 64 & 70 & 72 & 86 & 66 \\
\hline
\end{tabular}

${ }^{a}$ At a lag of six, the repeated word was always in the first and last serial positions.

${ }^{\mathrm{b}}$ Mean from lags 1 through 4 only. 
Table 3. Percentage of responses which were both correct and confident (rated " 1 ") as a function of lag and rate (ms/word), Experiment 1

\begin{tabular}{|c|c|c|c|c|c|c|}
\hline \multirow[b]{2}{*}{ Rate } & \multicolumn{6}{|c|}{. Lag } \\
\hline & 1 & 2 & 3 & 4 & $6^{\mathrm{a}}$ & $\operatorname{Mean}^{b}$ \\
\hline 117 & 2 & 5 & 10 & 15 & 62 & 8 \\
\hline 150 & 13 & 23 & 27 & 37 & 77 & 25 \\
\hline 183 & 30 & 37 & 48 & 37 & 62 & 43 \\
\hline 250 & 75 & 65 & 75 & 72 & 77 & 72 \\
\hline Mean & 30 & 38 & 49 & 40 & 69 & 44 \\
\hline
\end{tabular}

at a lag of six, the repeated word was always in the first and last serial positions.

${ }^{b}$ Mean of data from lags 1 through 4 only.

\section{Discussion}

This experiment indicates that repetition detection with confidence is difficult for rapid presentation rates-even when there are several intervening words between R1 and R2, and even when R1 and R2 are in different case. Furthermore, while repetition detection is above chance, ${ }^{2}$ it is difficult at presentation rates which have been shown to permit reliable recognition of unrepeated words (Potter, 1984).

Repetitions were easier to detect both at longer lags and slower presentation rates. Difficulty in detecting repetitions was most marked at the faster rates. For example, averaging across lags one through four at the rate of 117 ms, although subjects responded correctly on almost half of the trials, they responded correctly and with confidence on only $8 \%$ of trials. At the rate of $150 \mathrm{~ms}$, subjects were correct and confident on only $25 \%$ of trials, averaged across lags one through four, compared with $72 \%$ at the rate of $250 \mathrm{~ms}$.

The disparity betwcen accuracy and accuracy with confidence is in keeping with the fact that at the rapid rates most subjects reported that they rarely actually saw a repetition per se. Rather, it seems that the forced choice paradigm obliged them to exploit whatever intuitions were available. Most subjects were surprised to find out that their performance was even as high as it was.

Do lag and rate in fact have independent effects, or might lag and rate both influence performance by way of the same underlying variable: the

\footnotetext{
${ }^{2}$ The definition of chance here depends on whether token information, or only type information, is assumed to be involved. If randomly selected, two out of the eight list tokens would give the correct answer; only one of the seven list types would give the correct answer.
} 
elapsed time between the two instances? Figure 1 showing performance as a function of stimulus onset asynchrony (SOA) indicates that elapsed time alone does not fully predict performance. Although performance increases with SOA, almost completely nonoverlapping curves are obtained at different presentation rates. Hence, presentation rate affects performance apart from its correlation with SOA. Much the same pattern of results is observed if the R1-R2 interstimulus interval (ISI) is plotted. However, one caution should be noted. Presentation rate is confounded in this experiment with the presentation duration of both $\mathrm{R} 1$ and $\mathrm{R} 2$. It is possible that the main effect of rate might arise from differences in the duration of R1 and/or R2.

The different possible accounts of repetition blindness discussed in the introduction can now be evaluated (see Table 1). The Recognition Failure

Figure 1. Percentage correct (above) and percentage correct and confident (below) as a function of stimulus onset asynchrony, Experiment 1.

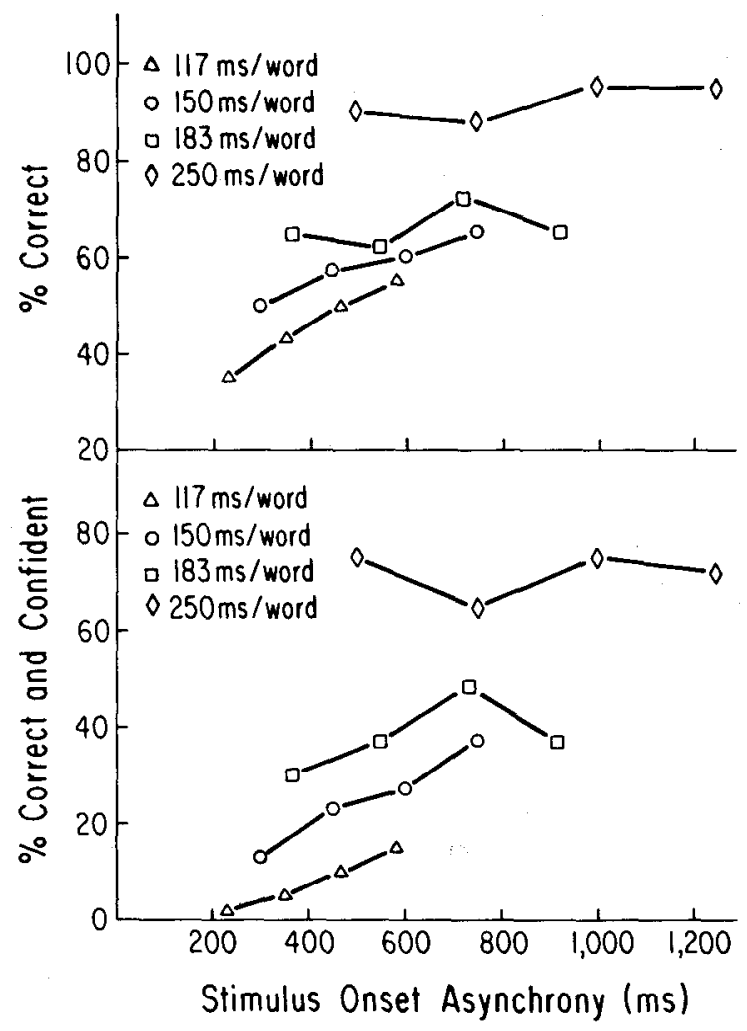


Hypothesis held that subjects fail to detect repetitions because they simply cannot recognize all the words in rapidly presented lists. Although not definitively ruled out by Experiment 1, this account is unlikely because subjects had considerable difficulty detecting repetitions even when words were presented for $150 \mathrm{~ms}$ apiece, which should allow easy recognition (Potter, 1984).

The Forgetting Hypothesis held that R1 is forgotten before R2 appears. This explanation can be ruled out by Experiment 1, because it would predict that repetition detection should be easier at shorter lags when there is not as much time to forget R1 before R2 appears. In contrast, in the current experiment subjects were better able to detect repetitions when more items intervened between $\mathrm{R} 1$ and $\mathrm{R} 2$.

Other explanations of repetition blindness remain possible, however. For example, the Multiple Comparisons Hypothesis holds that both words are seen but the viewer fails to realize they are the same word. This might happen if detecting a repetition entails performing a set of serial pairwise comparisons to determine which two list items are the same. If this is the case, these pairwise comparisons may take longer than the time per word allows. This possibility was tested by using a new task in Experiment 2, in which no such comparisons were required.

\section{Experiment 2}

Experiment 2 looked for evidence of repetition blindness in a different task. Subjects were asked to recall whole sentences which contained repeated words. Would subjects fail to detect or report repeated words, even when this would lead them to sacrifice the syntax and meaning of the sentence?

Pilot data suggested that it is the second instance (R2) which suffers in recall when a word is repeated. Hence, in the unrepeated control for this experiment, R2 was held constant but R1 was exchanged for a similar word which left the sentence meaning roughly the same. Recall of R2 was compared in the repeated and unrepeated conditions.

\section{Method}

\section{Subjects}

Thirty-six subjects participated, from the same pool previously described.

\section{Materials}

Nine sentences containing repeated words were written. In one control version of each sentence the first occurrence of the critical word (R1) was 
Table 4. Sample stimulus items in each condition, Experiment 2

\author{
Repeated: When she spilled the ink there was ink all over. \\ Unrepeated: When she spilled the liquid there was ink all over. \\ Blank: "When she spilled the ink there was all over.
}

replaced by another word, often a synonym, that preserved the general structure and meaning of the sentence (the unrepeated control). In the other control version, the second occurrence (R2) was omitted (the blank control); the resulting sentence was always ungrammatical or anomalous. The purpose of the blank control was to evaluate subjects' propensity to intrude R2 when it was not present. It also provided sentences which were missing a word, to encourage subjects to respond with ungrammatical sentences when they thought they saw them. Thus each sentence had three versions: one with repeated critical words, one with unrepeated critical words, and one with a blank in place of R2. The critical word pairs never included the first or last word of the sentence, and there were one to three intervening words. $\mathrm{Al}$ though some of the sentences were slightly awkward, all except those in the blank version were grammatically acceptable. The nine sentences and their controls are shown in the Appendix, and examples are shown in Table 4.

There were an additional 18 filler sentences in the experiment which were included to test another hypothesis. These fillers were randomly interspersed with the test sentences. None of the filler sentences contained repeated words.

\title{
Design and procedure
}

The three versions of each sentence (repeated, unrepeated, and blank appeared in different versions of the experiment, counterbalanced so that a given subject saw three sentences in each condition for a total of nine tes sentences per subject.

Each trial began when the subject pressed the space bar on the compute: keyboard. A row of asterisks appeared for $750 \mathrm{~ms}$ at the same location as thr subsequent words. Then the sentence appeared, one word at a time in thr same place. Each word was displayed for $117 \mathrm{~ms}$.

Subjects were instructed to read the sentence as carefully as possible an to recall the sentence out loud as soon as it ended. Subjects were warned tha some sentences would be strange or ungrammatical, and they were to repea them "as is", without "fixing them up". Three practice sentences precede the experimental sentences. 
Table 5. Percentage of trials on which $R 1$ and $R 2$ were recalled in each condition, Experiment 2

\begin{tabular}{lccc}
\hline Occurrence & Repeated & Unrepeated $^{\mathrm{a}}$ & Blank \\
\hline R1 & 90 & 94 & 92 \\
R2 & 22 & 79 & 0 \\
\hline
\end{tabular}

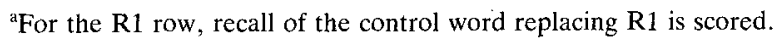

\section{Results}

Overall, performance on sentence recall was high. The number of trials in which subjects included R1 and R2 in their recall of the sentence was scored (see Table 5). It was almost always apparent from the serial position of the key word relative to other recalled words whether it was R1 or R2 that was recalled. When there was any question about this, the item was scored conservatively as an inclusion of R2.

The primary focus of Experiment 2 was recall of R2, shown in Table 5. Overall, subjects recalled R2 in $22 \%$ of repeated trials, $79 \%$ of unrepeated trials, and $0 \%$ of blank trials (i.e., R2 was never intruded). Analyses of variance by subjects and items were carried out on the number of correct recalls of R2 in the repeated and unrepeated conditions, omitting the blank condition. The overall Repeatedness effect was significant, $\min F^{\prime}(1,40)=$ $74.5, p<.0001$.

Although Experiment 2 was not designed to evaluate the effect of repetition on recall of R1 (because different words appear as R1 in the repeated and unrepeated conditions) it was still possible to score R1 and R1-control word recall. The results are shown in Table 5. There was little effect of a later repetition on recall of $\mathrm{R} 1$, which was recalled in $90 \%$ of repeated trials, $94 \%$ of unrepeated trials, and $92 \%$ of blank trials.

\section{Discussion}

Subjects in this experiment selectively omitted second instances of repeated words in sentence recall-even though the result was that the sentence became ungrammatical. The second instance of the repeated word (R2) was omitted on $78 \%$ of repeated trials and on $21 \%$ of unrepeated trials, and was never intruded on blank trials. Thus, a sentence environment is evidently not sufficient to save a repeated word from oblivion, even when sentence grammaticality is at stake.

It is clear that subjects treated the sentences as such, however, because 
overall recall was high. When subjects omitted R2 in the repeated condition, such omissions usually left the rest of the sentence largely intact (except for articles or other words adjacent to R2, which were sometimes omitted along with it). ${ }^{3}$ Responses in the repeated condition, but not the unrepeated condition, were usually ungrammatical, since subjects rarely intruded new words in place of the omitted R2.

What are the implications of these results for the hypotheses that have been suggested to account for repetition blindness? First consider the Recognition Failure Hypothesis, which attributed failure to detect repetitions to a general failure of recognition for all rapidly-presented words, repeated or not. This hypothesis is incorrect, since in Experiment 2 it was only repeated words, not unrepeated words, which suffered in recall.

Thus repetition blindness is due to a particular difficulty in processing second instances of repeated words. At what stage does this difficulty arise? Does R1 prevent the actual recognition of R2 (the Refractory Period Hypothesis), or is $\mathrm{R} 2$ recognized but not individuated as a distinct token from R1 (the Token Individuation Hypothesis)? Experiment 3 addressed this question by directly measuring the effect of a prior occurrence of a word on later recognition of that same word. If the Refractory Period Hypothesis is correct, we would expect a prior occurrence to inhibit later recognition of the same word. If instead the Token Individuation Hypothesis is correct, then we would expect a prior occurrence to have no effect or to benefit recognition of the same word a moment later.

\section{Experiment 3}

Experiment 2 demonstrated a marked failure to recall the second occurrence of a repeated word-even when this required sacrificing the meaning and grammaticality of the sentence. One might have expected a sentencc context to provide an ideal environment in which to stably encode recognized words. The fact that sentences did not preserve R2 might therefore imply that R2 was simply never recognized (the Refractory Period Hypothesis). As a direct test of this hypothesis, Experiment 3 measured near-threshold recognition of $\mathrm{R} 2$ in a situation similar to that in which repetition blindness occurs.

\footnotetext{
${ }^{3}$ Lower recall in the repeated condition cannot simply be due to the awkwardness of repeated sentences, because this ought to effect recall of the words following $\mathrm{R} 2$ just as much, and it does not. The word immediately following R2 was recalled in $66 \%$ of repeated trials, $72 \%$ of unrepeated trials, and $64 \%$ of blank trials. The second word after R2 was recalled in $95 \%$ of repeated trials, $92 \%$ of unrepeated trials, and $92 \%$ of blank trials. (The overall difference between first and second followers of R2 probably results from the fact that first followers were often short closed-class words, which are known to be more difticult to see in RSVP.)
} 
The task chosen was identification of the last words in lists that varied in length unpredictably. The last word in the list was presented briefly and followed by a mask. On half of the trials the target (last) word had also appeared earlier in the same list. If repetition blindness arises because there is a temporary elevation in recognition threshold for a repeated word, then one would expect lower accuracy in the repeated than the unrepeated condition. The time course of the effect on recognition threshold was investigated by varying the distance between the earlier instance of the target word (R1), and the target word itself (R2).

\section{Method}

\section{Subjects}

Eighteen subjects from the pool previously described participated in the experiment.

\section{Materials}

Test lists were made by modifying the lists in one of the versions of Experiment 1 (each list was truncated after R2). The lists therefore varied from four to eight words in length. In addition, two fillers of length two and one filler of length three were included to encourage subjects to attend to the whole list, by occasionally presenting a short list.

Each list occurred in two different conditions. "Repeated" trials contained the target word both earlier in the list and at the end. "Unrepeated" trials were the same except that another word was substituted for R1. Because these lists were taken from Experiment 1, in which lag was varied, there were six trials at each of five lags, 1, 2, 3, 4, and 6. This allowed us to investigate the possible effect of the lag between R1 and R2.

Pilot experiments indicated that determining which item in a rapid list was last was difficult when words occurred in mixed case, so words in this experiment were all presented in upper case.

\section{Design}

There were two versions of the experiment, each including 15 repeated trials and 15 unrepeated trials, plus the 3 fillers. The trials which were repeated in one version were unrepeated in the other version.

\section{Procedure}

Six trials preceded the main experiment. Each trial began when the subject pressed the space bar. A row of x's appeared for $500 \mathrm{~ms}$ at the same location as the subsequent words. Then the list appeared, one word at a lime in the 
same place. Each of the list words (excluding the last one) was displayed fo $117 \mathrm{~ms}$; the last (target) item was displayed for $67 \mathrm{~ms}$, and followed by a 11' ms row of percent signs, as a mask.

As soon as the list ended, subjects named the word they thought har appeared last in the list, guessing if they were unsure. They were instructer to answer as quickly and as accurately as possible. The accuracy of thei spoken response was measured.

\section{Results}

Subjects named the critical word correctly more often in the repeated condi tion $(59 \%)$ than in the unrepeated condition $(41 \%), \min F^{\prime}(1,38)=7.7$, , $<.01$. As can be seen in Table 6, there was no effect of lag on the percentag of correct responses to either repeated or unrepeated items. Although the were told about possible repetitions before the experiment began, few sut jects noticed the repeated words.

\section{Discussion}

Subjects are evidently more accurate at naming a briefly presented word they have seen it earlier in the list. This finding is inconsistent with th Refractory Period IIypothesis.

However, it is possible that the inproved performance in the repeate condition results from subjects guessing words that had appeared earlier i the list, which would sometimes be correct in the repeated condition (i.e when they guessed R1), but never in the unrepeated condition. This we checked by looking at how often subjects guessed unrepeated list words whic had occurred in the serial position of R1. This only happened once (less tha $1 \%$ ) in this experiment, so it is unlikely that pure guessing accounts for th repetition advantage. When subjects err in this task, most of their error

Table 6. Percent correct report of the last word as a function of R1-R2 lag, Expe1 ment 3

\begin{tabular}{lccccc}
\hline Condition & 1 & 2 & 3 & 4 & 6 \\
\hline Repeated & 67 & 59 & 50 & 65 & 52 \\
Unrepeated & 52 & 41 & 32 & 52 & 35 \\
Repeated-Unrepeated & 15 & 18 & 18 & 13 & 17 \\
\hline
\end{tabular}


consist of naming a word on the list, but it is usually the word preceding the target. This might suggest that it was difficult to determine which word occurred last, or that the next-to-last word was functionally last when the last word was imperceptible.

If repetition helps subjects to perceive the second instance of a word, then one might expect that in the unrepeated trials subjects would make more errors indicating partial perception of target words. There were 17 errors in which the subject gave a word similar to the target word, and all but one were in the unrepeated condition. This lends further credence to the conclusion that prior occurrences of the same word help recognition of the target word.

The fact that prior occurrences benefitted recognition of later occurrences of the same word is in one sense not surprising. This finding resembles the well-known effect of repetition priming. In a lexical decision task, for example, responses are faster to words which have already been presented earlier in the same test series (Scarborough, Cortese, \& Scarborough, 1977). This effect lasts from a lag of 0 (no intervening trials) to 30 trials, with no diminution in the size of the effect. Such insensitivity to lag was also found in Experiment 3. The range of time intervals between first and second occurrences of the same word in repetition priming studies is certainly longer than that used in the current experiments. Nevertheless, repetition priming does provide a precedent for the observation of a repetition benefit which does not diminish with lag.

Because words are easier to recognize when they are a repetition of an earlier word than when they are not, the Refractory Period Hypothesis must be false. The occurrence of R1 does not inhibit recognition of R2, so such inhibition cannot explain why subjects fail to detect repetitions (Experiment 1 ), and why subjects omit R2 in recall (Experiment 2). On the contrary, Experiment 3 suggests that $\mathrm{R} 2$ was probably recognized in these experiments. If sccond instances of repeated words are recognized, but can neither be recalled nor noticed as repetitions, it seems likely that they are simply not identified as distinct tokens. Thus repetition blindness must be blindness to the word as a distinct token, rather than blindness to the word itself. This is the Token Individuation Hypothesis. 


\section{General discussion}

Summary of results

Experiment 1

Subjects performed poorly at the task of detecting repeated words rapidly-presented lists, even when several words intervened between the $t$ instances of the repeated word, and even when one instance appeared capitals and the other appeared in lower case. Difficulty with this task, wh I call "repetition blindness", was most severe at the presentation rate of : ms/word, where subjects responded both correctly and confidently to $o$ $8 \%$ of the trials. Repetition detection was easier when greater lags separa the first and second occurrence of the repeated words. Thus, it is unlik that it is forgetting R1 before R2 appears (the Forgetting Hypothesis) wh accounts for repetition blindness.

\section{Experiment 2}

In a free recall task using sentences, it was found that subjects usur selectively omitted second instances of repeated words, but only rarely on ted those same words when the first instance had been changed so the st tence did not contain a repetition. Recall of the rest of the words in 1 sentence, including R1, was consistently high in both the repeated and un peated conditions.

Thus, providing a sentence context did not eliminate blindness for $t$ second of two identical words. As subjects performed very well in recall unrepeated words, repetition blindness cannot simply be the result of norn failure of perception and memory for any rapidly-presented words-repeat or not (the Recognition Failure Hypothesis). Furthermore, the difficulty detecting repetitions is not due to the difficulty of performing the many pa wise comparisons which may be entailed in repetition detection (the Multi] Comparisons Hypothesis), since no such comparisons needed to be made the recall task of Experiment 2.

\section{Experiment 3}

When the last word in a list was presented near threshold and the task $\mathrm{x}$ to name it, accuracy was higher when the word had also occurred earlier the list. The higher accuracy was not due to a guessing advantage. The res indicates that repetition aids recognition. So, repetition blindness must $n$ be blindness to R2 (the Refractory Period Hypothesis), but instead blindnc to the distinction between R2 and R1 (the Token Individuation Hypothesis)

The basic repetition blindness phenomenon is extremely robust and $h$ 
been replicated by now in nine other experiments. Many of these are described in Kanwisher (1986).

\section{Possible mechanisms for repetition blindness}

Figure 2 shows a model of visual information processing which can account for repetition blindness. The model makes a distinction between type information, about word identity, and token information about visual episodes. ${ }^{4}$ The two kinds of information are encoded in two distinct domains. As each new list word is recognized its type node becomes activated. In addition, token nodes are set up in parallel to the type nodes in order to encode the current episode, including the serial order of events and possibly other episodic information. After the type nodes have been activated and the token nodes have been established, links may be set up connecting the two. These links are depicted as pointers in the model, which assign a given activated type node to the appropriate token node. This process of token assignment will be called "token individuation".

Figure 2. A model depicting the integration of type and token information in the processing of visually presented lists.

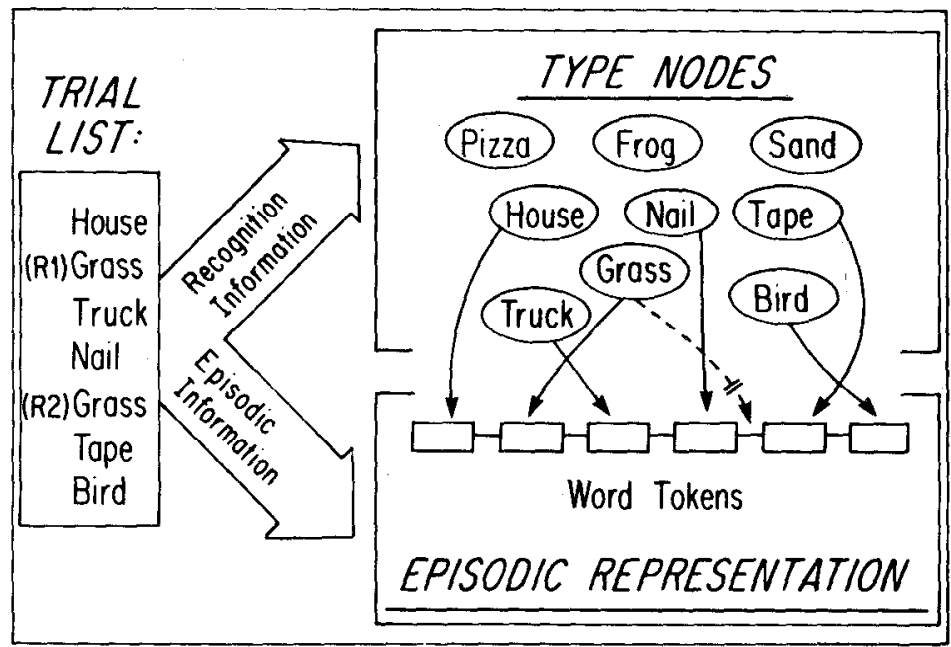

${ }^{4}$ A similar distinction has been proposed by Kahneman \& Ireisman (1984). 
The model accommodates repetition blindness as follows. Once a gives type node has been token individuated, it becomes unavailable for sub sequent (second) token individuation. This inhibition of second-token indi viduation does not influence further activation of the type node; it only in hibits assignment of the same type to a second token. Thus when R2 i presented, its type node will be activated but token individuation of R2 wi be inhibited. If no new token is assigned to R2, then encoding of R1 and R as separate events will be precluded, and the subject will only be aware $\mathrm{C}$ one occurrence of the repeated word.

The findings of Experiment 3 pose a challenge for the model: how wer subjects able to recall the target word when it was a repetition of an earlier occurring word? Presumably, it would have to be token individuated in orde to be recalled, yet it was a second token of a type. There are two possibl explanations of this result. First, subjects in the threshold recognition expes iment may have been able to token individuate R2 because they never ind: viduated $\mathrm{R} 1$ in the first place. If R1 was only recognized (which it must hav been, to boost $R 2$ recognition), and not individuated, then there would $b$ no inhibition to individuation of R2. This strategy would make sense, becaus subjects were never required to retain items from earlier in the list. Thus the may have performed the task by simply uncoupling type processing fror token processing, proceeding through the list by allowing type nodes to $b \epsilon$ come activated without individuating them. Then, when the list ended the would have been able to simply individuate the type which was just the becoming activated, in order to recall it. Further credence is given to thi interpretation by the fact that most subjects never noticed any repeate words, even though they were warned about them. This is what would $b$ expected if R1 was never token individuated. Thus, R2 may have been toke individuated because of failure to individuate R1. ${ }^{5}$

Second, even if R1 was individuated, it is possible that extra attention ma have been allocated to R2 in Experiment 3 (but not in Experiments 1 an 2 ), and that this might have enabled subjects to override the inhibition $t$ token individuation of R2. However, this possibility does not reduce th relevance of the findings of Experiment 3 for the first two experiments, $i$ which extra attention could not be allocated to R2. The important finding $c$ Experiment 3 is not that $\mathrm{R} 2$ can be recognized when subjects try hard to se it, but instead that such recognition is helped, rather than hindered, by a earlier occurrence of the same word. If earlier occurrences can only hel

\footnotetext{
${ }^{5}$ This hypothesis will be tested in future by simply including memory instructions for all list items in threshold task like that of Experiment 3. Subjects would first name the target (last) word, and then give many other words as possible. Presumably, retention of list items for later recall will require token indivic ation of each one. According to this hypothesis, then, such memory instructions would be expected to decre: the subject's ability to individuate repeated targets, and target recall should suffer more for repeated targ than unrepeated ones.
} 
recognition, there is every reason to suspect that R2 was recognized in Experiments 1 and 2 .

The model accommodates the following findings: (1) The difficulty of recalling or detecting second instances of repeated words; (2) the fact that repeated words are not more difficult to recognize in a threshold task than unrepeated words, (and-further--the fact that words actually benefit from such repetition); and (3) the fact that repetition blindness occurs at presentation rates which allow encoding of token information about nonrepeated words in sentences. Insofar as recall of serial order indicates that an episodic representation has been established, the propensity to recall sentences in order provides further evidence that token information for nonrepeated words was available.

The repetition benefit observed on threshold word recognition in Experiment 3 is also what would be expected according to the model. Whether R1 and $\mathrm{R} 2$ are individuated as separate events or not, the activation from both stimuli should be summed in the type node. This would increase the activation of the type node, boosting recognition. The repetition benefit observed is also consistent with the literature on repetition priming (Scarborough, Cortese, \& Scarborough, 1977).

Further findings can be accounted for naturally in this model. First, the effects of rate and lag fit well with the idea that there is a period after a given type has bcen token individuated, during which it cannot be individuated again. One might expect this inhibition to diminish with time, and indeed Experiment 1 showed that longer R1-R2 intervals result in better repetition detection. The effect of lag (as separate from SOA) may result from the fact that the decay of inhibition of R2 individuation is hastened by the individuation of other type activations.

The distinction made here between type and token information is reminiscent of similar distinctions made in the past by others. It might, for example, be thought of as a visual perceptual analogue to Tulving's (1972) distinction between semantic and episodic memory. More recently, Salasoo, Shiffrin, and Feustel (1985) have suggested a model of the memory processes underlying threshold identification of words and pseudowords. They contrast "codification", or the establishment of permanent traces for words or repeated pseudowords, with repetition effects which decay over time and are contextdependent or "episodic". The codified trace, which "responds ... to a set of features and serves to label, code, name, or identify those features (p. 51)" behaves much like the type nodes described above. The storage of particular context-dependent episodic images, on the other hand, resembles the tokens in the current model. Salasoo et al. describe each "code as functionally discrete from the episodes relating to it (p. 73)", much as the current model distinguishes type activations from token slots. Further parallels between repetition blindness and other phenomena will be discussed in the final sec- 
tion after considering what role repetition blindness may play in visual perception.

\section{The significance of repetition blindness}

The limitation on the rate at which two tokens of the same type can be individuated could be regarded simply as a shortcoming of the system, or instead it could be a design feature of the visual system which allows a useful interpretation of visual events. An independent question is whether repetition blindness is best understood as a specifically linguistic (verbal) phenomenon, or as an aspect of visual perception in general. In order to address the first question, therefore, the possible processing utility of repetition blindness will be considered both in the context of language and in the context of general visual processing.

I shall argue that any potential processing utility of repetition blindness would be nonlinguistic because (1) it is hard to imagine any useful role repetition blindness might have in linguistic processing, and (2) repetition blindness exhibits little sensitivity to linguistic parameters. Since repetition blindness does not occur in audition (Kanwisher, 1986, Kanwisher \& Potter, 1987) ${ }^{6}$ and does not occur at presentation rates typical of normal speech and reading (Experiment 1), ${ }^{7}$ it seems unlikely that it could play any role in normal linguistic processing. More importantly, the distinction between different tokens of the same type is crucial in language, and it is hard to see how the inability to individuate such tokens could ever be advantageous.

If repetition blindness played some particular role in linguistic processing, one might expect it to exhibit some sensitivity to linguistic parameters-which it does not (Kanwisher, 1986). On the contrary, repetition blindness occurs for both open and closed class words, and it occurs to the same degree whether or not one instance of the repeated word occurs as part of a compound noun phrase (e.g., "Sailors in bars discuss sand bars which are dangerous", versus "Sailors in bars discuss city bars which are dangerous"). In addition, repetition blindness only affects repeated words embedded in sentences-not repeated meanings (e.g., "couch ... couch" shows repetition blindness but "sofa ... couch" does not). Finally, repetition blindness causes a grammatical sentence to be judged as ungrammatical, showing that syntactic

\footnotetext{
${ }^{6}$ This experiment used compressed speech to match auditory and visual presentation rates. While percentage correct recall of R2 in unrepeated sentences was similar in RSVP and compressed speech, recall of R2 in repeated sentences fell only in the RSVP, and not the compressed speech, version of the experiment.

${ }^{7} \mathrm{R} 2$ is recalled just as well in repeated sentences as in unrepeated sentences, when the sentences are presented in RSVP at a rate of 4 words per second (Kanwisher, 1986).
} 
analysis occurs later in processing than the process responsible for repetition blindness (Kanwisher, 1986, 1987). ${ }^{8}$ Together, these results indicate that repctition blindness probably occurs at the level of the encoding of recognized words, rather than within the linguistic processor. Thus if there are any possible processing advantages of repetition blindness, they are likely to be found for vision in general, not for linguistic processing in particular.

The ability to distinguish between different tokens of the same type is crucial to both language and vision. However, there are some common situations where the failure to assign distinct token identities to two events of the same type might have certain advantages. Suppose that the visual information received about an object is fragmented by intermittencies either in visual stimulation (e.g., brief occlusions) or in internal processes (e.g., saccades). Treating the resulting fragments of information as the same token would allow the perceiver to maintain a continuous representation of enduring objects in the visual world, despite the interruptions--just as visual temporal summation allows perceivers to maintain a continuous representation of visual form despite still briefer interruptions such as blinks. It remains to be investigated whether the failure to individuate tokens at rapid presentation rates plays a role in bridging the information obtained from successive eye fixations or successive views of the same object.

Repetition blindness is of interest whether or not it turns out to have any such useful function in processing. The phenomenon is a case in which typeactivation is uncoupled from the process which normally anchors that activation to an episodic (token) representation of an event. How the token representation is related to short-term memory, and whether attention is required to anchor types to episodic tokens, remains to be discovered.

\section{Repetition blindness and other repetition phenomena}

\section{Apparent motion}

Apparent motion is perceived when two stimuli appear sequentially in nearby locations, provided that the temporal disparity between their occurrences falls within a certain range. This temporal range is roughly the same as that within which repetition blindness is observed. One might suppose that

\footnotetext{
${ }^{8}$ This experiment used naming latency to a word immediately following a repeated word in a sentence, together with judgments of the grammaticality of the sentence, to determine whether the second instance of a repeated word is registered by the parser. Sentences whose grammaticality hinged on inclusion of the second instance were rated as grammatical less often than those same sentences when they appeared in an unrepeated condition. RTs mirrored this effect, but did not reach significance. These results suggest that the parser often does not "see" the second instance of a repeated word.
} 
apparent motion is simply the spurious assignment of one token identity to the two different stimuli, like repetition blindness. However, instead of failing to sce one of the two occurrences, the observer perceives a singlc moving object. In further contrast with repetition blindness, apparent motion seems to occur "between any two shapes having the proper spatial and temporal characteristics, irrespective of their identity (Kolers, 1972)". Thus, apparent motion is different from repetition blindness in that it does not require type information, although type identity does enhance motion under some conditions. Nevertheless, the construal of apparent motion as an error in token identity assignment is interestingly similar to the interpretation offered for repetition blindness.

\section{The Ranschburg effect}

Ranschburg (as reported by Jahnke, 1969) discovered that when subjects viewed a sequence of six digits, their ordered report of the digits was better when the sequence did not contain any repeated items. Numerous possible sources of the effect have been suggested. Jahnke (1972) showed that the Ranschburg effect is completely eliminated when interserial repetitions-that is, items which occur in more than one list-are eliminated. Thus, he suggests that proactive interference is a major factor in the Ranschburg effect. Another study (Hinrichs, Mewaldt, \& Redding, 1973) suggests that the Ranschburg effect arises from the inappropriate application of a guessing strategy in which subjects simply keep track of the digits not mentioned in a list, and recall the others. Crowder (1968) suggested the importance of output phenomena in the genesis of the Ranschburg effect, because he found that the effect could arise when subjects were instructed to prefix their response with a standard memorized sequence. Items within the prefix inhibited subsequent recall of identical items on the main list. Harris and Jahnke (1972) also argued that the Ranschburg effect occurs at least partly during response output, since when they instructed subjects to omit the item in serial position two from their recall, there was no deficit in recall of that same item appearing later in the same list.

Hence, there is little consensus on the explanation of the Ranschburg effect. Although there may be several different factors involved, there does seem to be a core phenomenon involving a recall deficit for repeated word: in situations where subjects fail to notice repetitions of words. This is proba bly related to repetition blindness in that both concern the difficulty of encod ing and retaining distinct tokens of the same type, but the similarity end: there. The differences include the following: (a) The Ranschburg effect oc curs at a wide range of presentation rates; and (b) it is found for both auditor: or visual stimuli. In contrast, repetition blindness only occurs with visua 
presentation (Kanwisher \& Potter, 1987), and only occurs when words are presented at a rate of 6 items per second or more (Kanwisher, 1986). (c) While the Ranschburg cffect requires interserial repetitions, repetition blindness does not. (d) While the Ranschburg effect is decreased when the repeated words are closer together in a list, the opposite is found for repetition blindness. (e) Response output phenomena, which might explain the Ranschburg effect, cannot explain the results of Experiment 1, the fact that repetition blindness does not occur for auditorily presented stimuli, or the elimination of repetition blindness at slow rates. (f) Finally, the Ranschburg effect is much smaller in magnitude than repetition blindness. Thus, not only are there many differences between repetition blindness and the Ranschburg effect, but none of the proposed explanations of the Ranschburg effect can account for the current findings about repetition blindness.

\section{Typing errors}

In a study of skilled typing, Rumelhart and Norman (1982) propose a model for the control of the hands and fingers during typing. They report that "doubling" errors (such as "scrren" for "screen", and "liitle" for "little") are produced by their model and by skilled typists. To account for these errors, they propose that "the model has only 'type' schemata-no 'token' schemata-with only a weak binding between the special schema that signals a doubling, and its argument". While the form of their doubling errors is not strictly parallel to repetition blindness, Rumelhart and Norman's work (1982) suggests that the distinction between type and token information is common to both perception and motor control.

\section{Masked repetition priming}

Forster and Davis (1984) have demonstrated repetition priming in a lexical decision task using primes (which were displayed for $60 \mathrm{~ms}$, immediately preceding the target, and which differed from the target only in letter case). Forster and Davis argue that since subjects could not report the identities of primes, the repetition effects obtained could not be episodically mediated, but must be "an automatic consequence of repeated access of the same lexical entry".

An alternative account would be that prime and target were simply never token individuated, and hence were treated like a single stimulus. Forster and Davis' failure to find masked priming effects for nonwords does not contradict this interpretation, because there is no processing locus where information from a repeated nonword and target could fuse together: nonwords do not have type nodes at the word level, and letter-level masked repetition priming was shown not to occur (Forster \& Davis, Experiment 2). 
Hence Forster and Davis' findings are consistent with the idea that maskec repetition primes lower reaction times to their targets because subjects dc not token individuate them as separate items in the first place. Notice, how ever, that on this account "masked repetition priming" may be a misnome: because it does not involve "repeated access of the same lexical entry".

\section{Very short term memory}

Based on experiments in which subjects are asked to freely recall entir RSVP word lists, Potter (1983) has proposed a distinction between short tern memory (STM) and a more labile "very short term memory" (VSTM). Sub jects can rarely report more than 2 or 3 words from a rapid 5- or 6-word list even when it is presented at rates which ought to allow easy recognition o all the words. Potter has suggested that incoming words are recognized an fleetingly registered in VSTM, but will be quickly forgotten unless they be come more stably encoded in STM. VSTM is not organized in an ordered phonological form like STM; the suggestion here is that it may simply consis of untokenized (in this case, lexical) type activations. According to this view storing items in STM might entail (or be identical to) token individuation. 1 so, then one might expect repetition blindness to depend on STM encodin of $\mathrm{R} 1$, as suggested in footnote 5 above.

\section{Perception of recency and frequency}

While repetition blindness occurs because subjects fail to individuate rer ognition tokens at rapid rates of list presentation, subjects are surprising] good at both identifying and remembering distinct tokens of the same typ when they are presented at slower rates. Hintzman and Block (1971) pri sented subjects with a list of 50 words, which included some repetition Subjects were accurate at subsequently judging the list locations of the worc and had no problem independently locating both list positions of repeate words. Their subjects could also notice and remember separately informatic about the frequency of occurrence of words presented in two different list Thus, repetition blindness is not an instance of a general difficulty peop have in keeping track of distinct tokens of the same type, but is rather t1 failure at high rates of a system which works impressively well at norm presentation rates.

\section{Illusory conjunctions and visual dissociation}

Illusory conjunctions (Treisman \& Schmidt, 1982) and visual dissociatic (Intraub, 1985) result when several items are displayed slowly enough allow identification of components or features of each item, but too rapid to allow determination of which components or features are part of the san 
visual event. The result is that the features or identities of items in the display are combined erroneously. In cases of illusory conjunction, for example, subjects may report a green $\mathrm{X}$ as a pink $\mathrm{X}$, when it appears near a pink $\mathrm{O}$ in a display. Visual dissociation, on the other hand, occurs when subjects view a rapid series of pictures of simple objects and are asked to report which pictured object has a frame around it. The frame often perceptually "migrates" away from the picture it actually surrounded, such that subjects are convinced that they saw the frame around a different picture.

Illusory conjunctions and visual dissociation resemble repetition blindness in that all three result from the incorrect assignment of types (or featuretypes) to visual tokens. While repetition blindness results from a failure to assign two different occurrences of the same type to two different tokens, these other phenomena result from a failure to correctly assign two different visual types (e.g., green and $X$, or chair and frame) to the same token. Thus repetition blindness, illusory conjunctions, and visual dissociation can all be regarded as failures of token assignment.

\section{Conclusion}

Subjects have been shown to have difficulty detecting or recalling repetitions of words in rapidly-presented lists and sentences, even when the two occurrences of the repeated word are nonconsecutive and differ in case. Repetition blindness is robust enough that subjects will omit a repeated word in recall of a sentence, even at the sacrifice of grammaticality and meaning. These and the other findings of the present study have been explained in terms of a processing distinction between identifying words as types and individuating them as episodic tokens of those types. The fact that words can be type-identified without being token-individuated demonstrates an important dissociation in visual processing. Although the correct establishment of links between visual types and visual tokens must be crucial in visual perception, the failure at high rates to assign a separate token identity to the second occurrence of an item may serve a useful role in maintaining the continuity of perceived events despite intermittencies in the available information. 


\section{Appendix}

Stimulus materials, Experiment 2; the words used in the unrepeated condition are shown in parentheses

1. The brown couch (sofa) and black couch were stolen.

2. To use (a) radio (the headphones) the radio must have batteries.

3. We asked for water (wine) although water was unavailable (available)

4. When she spilled the ink (liquid) there was ink all over.

5. We got into this van (vehicle) and another van for the commute.

6. His collection of books (things) will include more books about travel.

7. It was work (day) time so work had to get done.

8. Her jacket was red (pink) because red is conspicuous.

9. We were eating (dining) although eating was unnecessary.

\section{References}

Crowder, R.G. (1968). Repetition effects in immediate memory when there are no repeated elements in $t$ stimuli. Journal of Experirriental Psychology, 78, 605-609.

Forster, K.I., \& Davis, C. (1984). Repetition priming and frequency attenuation in lexical access. Journal Experimental Psychology: Learning, Memory, and Cognition, 10, 680-698.

Gilinsky, A.S. (1968). Orientation-specific effects of patterns of adaptation light on visual acuity. Journal the Optical Society of America, 58, 13-18.

Harris, C., \& Jahnke, J.C. (1972). Effect of partial recall on the Ranschburg phenomenon. Journal of Exl rimental Psychology, 93, 118-122.

Hinrichs, J.V., Mewaldt, S.P., \& Redding, J. (1973). The Ranschburg effect: repetition and guessing short-term memory. Journal of Verbal Learning and Verbal Behavior, 12, 64-75.

Hintzman, D.L., \& Block, R.A. (1971). Repetition and memory: Evidence for a multiple-trace hypothes Journal of Experimental Psychology, 88, 297-306.

Intraub, H. (1985). Visual dissociation: An illusory conjunction of pictures and forms. Journal of Experimen Psychology: Human Perception and Performance, 11, 431-442.

Jahnke, J.C. (1969). The Ranschburg Effect. Psychological Review, 76, 592-605.

Jahnke, J.C. (1972). The effects of intraserial and interserial repetition on recall. Journal of Verbal Learn and Verbal Behavior, 11, 706-716.

Kahneman, D., \& Treisman, A. (1984). Changing views of attention and automaticity. In Parasuraman, R., Davies, D.R. (Eds.), Varieties of attention. New York: Academic Press.

Kanwisher, N.G. (1986), Repetition blindness: Type recognition without token individuation. Unpublisł doctoral dissertation, Massachusetts Institute of Technology, Cambridge.

Kanwisher, N.G., \& Potter, M.C. (1987). Visual types and visual tokens. Manuscript submitted for publi tion.

Kanwisher, N.G. (1987). Repetition blindness and grammaticality judgements. Manuscript in preparation Kolers, P.A. (1972). Motion perception. Oxford: Pergamon Press.

Norman, D.A. (1986). Reflections on cognition and parallel distributed processing. In McClelland, J. Rumelhart, D.E., \& the PDP Research Group, Parallel distributed processing: Explorations in microstructure of cognition, Vol. 2: Psychological and biological models, Cambridge: MIT Press. 
Potter, M.C. (1983). Representational buffers: The eye-mind hypothesis in picture perception, reading, and visual search. In Rayner, K. (Ed.), Eye movements in reading: perceptual and language processes, New York: Academic Press.

Potter, M.C. (1984). Rapid serial visual presentation (RSVP): A method for studying language processing. In D.E. Kieras \& M.A. Just (Eds.), New methods in reading comprehension research. NJ: Hillsdale.

Rumelhart, D.E., \& Norman, D.A. (1982). Simulating a skilled typist: A study of skilled cognitive-motor performance. Cognitive Science, 6, 1-36.

Salasoo, A., Shiffrin, R.M., \& Feustel, T.C. (1985). Building permanent memory codes: Codification and repetition effects in word identification. Journal of Experimental Psychology: General, 114, 50-77.

Scarborough, D.L., Cortese, C., \& Scarborough, H.S. (1977). Frequency and repetition effects in lexical memory. Journal of Experimental Psychology: Human Perception and Performance, 3, 1-17.

Treisman, A., \& Schmidt, H. (1982). Illusory conjunctions in the perception of objects. Cognitive Psychology, $14,107-141$.

Tulving, E. (1972). Episodic and semantic memory. In E. Tulving \& W. Donaldson (Eds.), Organization of memory. New York: Academic Press.

Résumé

'Irois expériences sont décrites qui, grâce à ta technique RSVP (Présentation séquentielle visuelle rapide), permettent de mettre en évidence un nouveau phénomène cognitif appelé "cécité à la répétition". Des sujets éprouvent des difficultés à détecter des mots répétés-même lorsque les deux occurrences sont non consécutives et diffèrent par leur cas (Expérience 1). Lorsqu'on demande aux sujets de répéter immédiatement des phrases (Expérience 2), ils oublient sélectivement la deuxième occurrence du mot répété, sacrifiant le sens et la grammaticalité de la phrase. Dans l'expérience 3, le seuil de reconnaissance pour le dernier mot d'une liste était plus élevé et non plus faible lorsque ce mot était déjà apparu dans la même liste. La cécité à la répétition ne résulte donc pas d'une période réfractaire pour la reconnaissance des secondes occurrences de mots. Ces résultats appuient une distinction entre les processus perceptuels de (1) reconnaissance d'un mot comme étant d'un certain type et (2) d'identification d'un mot comme un token particulier de ce type. La cécité de répétition a lieu lorsque des mots sont reconnus en tant que types mais ne sont pas identifiés en tant que tokens. 